\title{
Effect of Non-Integer Delay on Ranging Accuracy for Ultra-Reliable Systems
}

\author{
Mohammad Soliman, Stephan Sand, Martin Schmidhammer and Emanuel Staudinger \\ German Aerospace Center (DLR) \\ Institute of Communications and Navigation \\ Oberpfaffenhofen, 82234 Wessling, Germany \\ Email: \{mohammad.soliman, stephan.sand, martin.schmidhammer, emanuel.staudinger\}@dlr.de
}

\begin{abstract}
Ultra-reliable communication systems are drawing a lot of attention due to the rising demand on new wireless technologies for safety critical applications. Many of these applications require ultra-reliable distance estimation between the communicating nodes. Automatic coupling between train wagons is one of the scenarios where ultra-reliable communication and ranging at short distances is required. The main objective of this paper is to define a theoretical channel model for the aforementioned scenario, to define a proper discrete equivalence of the communication system model, and to derive Cramér Rao Lower Bounds for ranging accuracy. Ranging accuracy simulation results are provided using three systems: ITS-G5, IRUWB, and a proposed 5G wide band system operating in the mm-Wave frequency band. We show from the results that the proposed $\mathrm{mm}$-Wave system is suitable for ultra-reliable ranging at short distances.
\end{abstract}

Index Terms-ranging, CRLB, ultra-reliable, delay estimation, mm-Wave, 5G

\section{INTRODUCTION}

With the advance of telecommunications towards $5 \mathrm{G}$ wireless technologies, the span of applications increases from the classical voice and data communications using smart phones to machine-type and mission critical communications. Ultrareliable communication systems are of special interest due to the rising amount of mission critical applications requiring high reliability levels [1]. These applications include vehicleto-vehicle $(\mathrm{V} 2 \mathrm{~V})$ communications for cars, trains, and other moving vehicles and machine-to-machine (M2M) communications in various environments such as factories, smart homes, and offices.

The aforementioned applications as well as other applications require ultra-reliable communications and distance estimation between two communicating nodes [2]. For example, automatic coupling in the railway domain requires ultra-reliable distance estimation between connecting (or disengaging) train wagons. The same requirements apply for car platooning, robots communicating in factories, and many others.

Distance estimation between two communicating nodes requires accurate delay estimation of the line-of-sight (LOS) signal being transmitted from one node to the other. The delay estimation accuracy depends on the strength of the LOS component, effect of the channel on the signal, system bandwidth, and other factors such as hardware impairments and Doppler caused by motion [3].

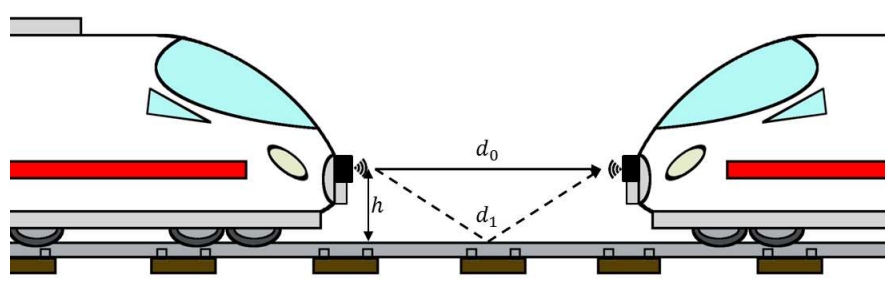

Fig. 1: Representation of a two-path channel model between two train wagons.

Impulse radio-ultra wide band (IR-UWB) systems were thought of as good candidates for ranging applications in short-range scenarios and delay estimation accuracy of such systems were studied extensively in literature in the last two decades [4]-[7]. Due to the regulations of emission limits to prevent interference with primary applications, a new spectrum candidate was demanded for $5 \mathrm{G}$ applications. Millimeter-Wave (mm-Wave) frequency bands were chosen as attractive candidates for new wide band standards including the IEEE 802.15 Task Group 3c (IEEE 802.15.3c) [9] and IEEE 802.11ad Task Group [10] standards. mm-Wave wide band systems were also studied for ranging applications [2], [11].

In this paper we focus on ranging estimation in the application of automatic coupling between train wagons at short distances. To accurately analyze the ranging accuracy, we define a realistic two-path channel model and derive Cramér Rao Lower Bounds (CRLB) using the proposed channel. We use the bounds to compare the ranging performance of three candidate communication systems. The three systems we choose to compare between in this paper are the Intelligent Transport Systems operating in the $5 \mathrm{GHz}$ frequency band (ITS-G5), IR-UWB based system, and a proposed 5G mmWave system.

\section{Channel Model}

As visualized in Figure 1, one transceiver node is connected to the nose of each train wagon. The heights of the transceivers to the ground are known. The transceivers are assumed to be connected with directional antennas having known directivity and gain characteristics to decrease multipath and in general 


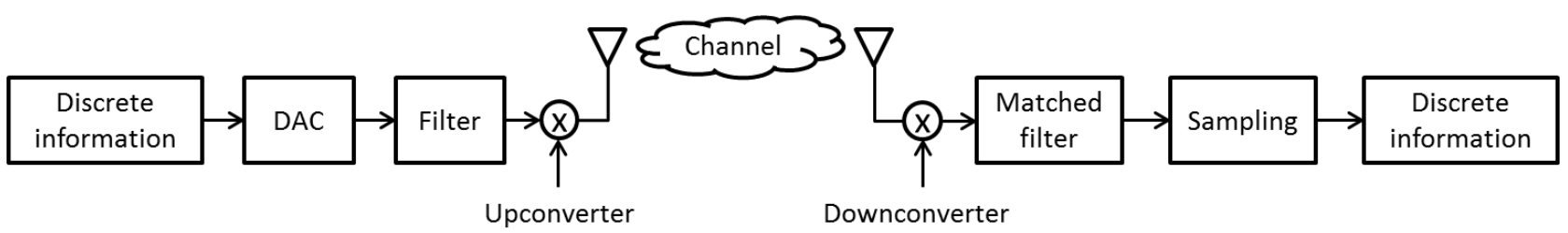

(a)

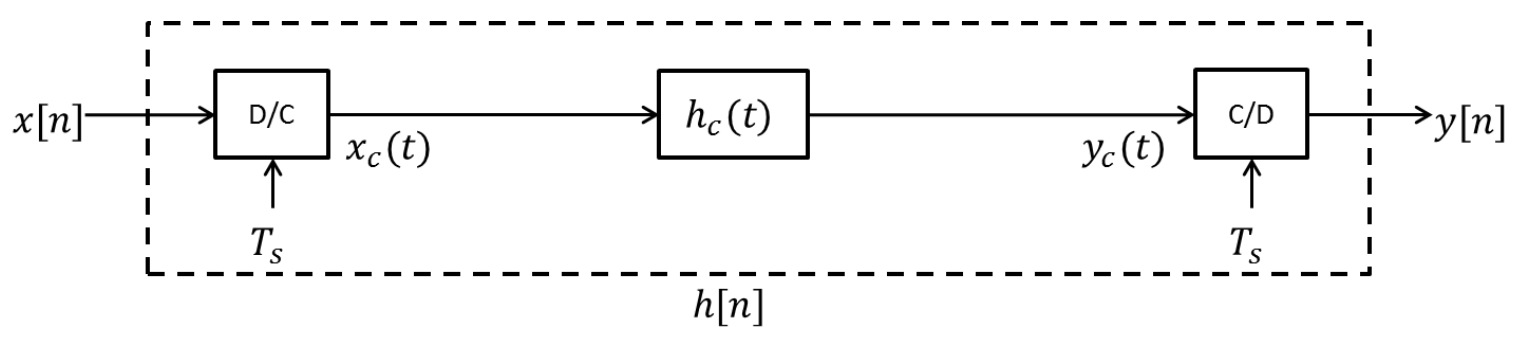

(b)

Fig. 2: System representation. (a) Block diagram of the transmitting and receiving nodes. (b) An equivalent discrete representation of the system in (a).

influence from the environment. In this specific scenario, it is assumed that the strongest received components are the LOS signal and a reflected signal from the ground. A good statistical approximation is the two-path channel model [8].

The two-path channel model assumes a superimposed signal consisting of a LOS component and a slightly delayed ground reflected component. The reflected component has a lower amplitude due to reflection loss and lower directive antenna gain but introduces varying constructive and destructive interference effects due to the phase difference with the LOS component. Depending on the geometry, the two received signals might overlap, resulting in a stretched distorted pulse at the receiver side that might cause variation to the distance estimation.

The statistical channel response $h_{c}(t)$ is expressed as follows:

$$
h_{c}(t)=a_{0} \delta\left(t-\tau_{0}\right) \mathrm{e}^{-\phi\left(\tau_{0}\right)}+a_{1} \delta\left(t-\tau_{1}\right) \mathrm{e}^{-\phi\left(\tau_{1}\right)}
$$

where $\tau_{0}=d_{0} / c$ and $\tau_{1}=d_{1} / c$ are the propagation delays of the LOS and reflected signals. $c, d_{0}$ and $d_{1}$ are the speed of light, the traveling distance of the LOS path, and that of the reflected path, respectively. The distance $d_{1}$ is a function of $d_{0}$ with the relation $d_{1}=\sqrt{(2 h)^{2}+d_{0}^{2}}$. The phases of the received signals are denoted as $\phi\left(\tau_{0}\right)$ and $\phi\left(\tau_{1}\right)$ and are delay dependent with the relation $\phi\left(\tau_{i}\right)=j 2 \pi f_{c} \tau_{i}$ for $i=0,1$. $f_{c}=c / \lambda$ is the carrier frequency.

The gains of the LOS path and the reflected path are

$$
\begin{aligned}
& a_{0}=\sqrt{G_{\text {ant,LOS }}}\left(\frac{\lambda}{4 \pi d_{0}}\right), \\
& a_{1}=\sqrt{G_{\text {ant,ref }}} \Gamma(\theta)\left(\frac{\lambda}{4 \pi d_{1}}\right) .
\end{aligned}
$$

$G_{\text {ant,LOS }}$ and $G_{\text {ant,ref }}$ are the antenna gains of the LOS and reflected paths. The coefficient $\Gamma(\theta)$ is the loss due to ground reflection.

\section{SySTEM MODEL}

Figure 2(a) describes the system design. It should be noted that the channel is convoluted with the signal in the continuous domain while the information being processed in the receiver is discrete. The discrete information is used for synchronization, equalization, and further data processing. To represent the discrete received signal, a sampled equivalence of the channel response written in (1) can be directly used:

$y[n]=a_{0} s\left[n-\frac{\tau_{0}}{T_{s}}\right] \mathrm{e}^{-\phi\left(\tau_{0}\right)}+a_{1} s\left[n-\frac{\tau_{1}}{T_{s}}\right] \mathrm{e}^{-\phi\left(\tau_{1}\right)}+w[n]$,

where $T_{s}$ is the sampling time, $s[n]$ is the discrete equivalent to the transmitted pulse and $w[n]$ is additive white Gaussian noise (AWGN) with zero mean and variance $\mathrm{E}\left\{w^{2}\right\}=$ $\sigma^{2}$. This representation is only valid if the values $\tau_{0} / T_{s}$ and $\tau_{1} / T_{s}$ are integers because the sequence $s[n]$ is only defined by integer values $n$. This is not a realistic representation and it is specifically not valid with the proposed channel model because the proposed channel is geometry based and the delay should take any non-integer value based on the distance between the transceivers.

An equivalent discrete channel model to $h_{c}(t)$ should be accurately derived to take non-integer delays into account. Figure 2(b) describes the block diagram representation of the equivalent system. $h[n]$ can be represented as [12]:

$$
\begin{aligned}
h[n]= & a_{0} \operatorname{sinc}\left(t-\tau_{0}\right) \mathrm{e}^{-\phi\left(\tau_{0}\right)} \\
& \quad+\left.a_{1} \operatorname{sinc}\left(t-\tau_{1}\right) \mathrm{e}^{-\phi\left(\tau_{1}\right)}\right|_{t=n T_{s}}
\end{aligned}
$$


and the discrete received signal $y[n]$ is properly represented as

$$
\begin{aligned}
y[n]=a_{0} s[n] * \operatorname{sinc}\left(n-\tau_{0} / T_{s}\right) \mathrm{e}^{-\phi\left(\tau_{0}\right)} & \\
& +a_{1} s[n] * \operatorname{sinc}\left(n-\tau_{1} / T_{s}\right) \mathrm{e}^{-\phi\left(\tau_{1}\right)}+w[n]
\end{aligned}
$$

where $(*)$ represents the convolution operation. Using this representation we can focus on the equivalent effect of the continuous time system while only dealing with discrete transmitted and received data.

\section{CRAmÉr RAO Lower Bounds (CRLB) FOR DisTANCE ESTIMATION}

The CRLB is a fundamental concept in estimation theory and is widely used to estimate the minimal achievable error variance for an unbiased estimator. It must be noted that this performance is typically not achievable by practical estimators.

Using the received signal represented in (5), we are interested in estimating the parameters $\overline{\boldsymbol{\alpha}}=\left[\tau_{0}, \tau_{1}\right]^{\mathrm{T}}$. To do so, we consider that we are observing $m$ measurement samples and we construct a joint probability density function (pdf) over all samples with respect to the parameters we are interested to estimate

$$
\mathrm{p}(\overline{\boldsymbol{y}} \mid \overline{\boldsymbol{\alpha}})=\prod_{i=1}^{m} \mathrm{p}\left(y_{i} \mid \overline{\boldsymbol{\alpha}}\right)=\left(2 \pi \sigma^{2}\right)^{-\frac{m}{2}} \mathrm{e}^{-\frac{1}{2 \sigma^{2}} \sum_{i=1}^{m}\left|s_{i}(\overline{\boldsymbol{\alpha}})-y_{i}\right|^{2}}
$$

where

$$
\begin{aligned}
s_{i}(\overline{\boldsymbol{\alpha}})=a_{0} s[i] * \operatorname{sinc}\left(i-\tau_{0} / T_{s}\right) \mathrm{e}^{-\phi\left(\tau_{0}\right)} & \\
& +a_{1} s[i] * \operatorname{sinc}\left(i-\tau_{1} / T_{s}\right) \mathrm{e}^{-\phi\left(\tau_{1}\right)} .
\end{aligned}
$$

The log-likelihood can be easily constructed by calculating the natural logarithm of (6). The CRLB of the parameters in $\bar{\alpha}$ are the diagonal elements of the inverse of the $2 \times 2$ Fisher Information Matrix (FIM) $\boldsymbol{F}(\overline{\boldsymbol{\alpha}})$ given by

$$
\begin{aligned}
{[\boldsymbol{F}(\overline{\boldsymbol{\alpha}})]_{k, k} } & =\frac{2}{\sigma^{2}} \sum_{i=1}^{m}\left|\frac{\partial}{\partial \alpha_{k}} s_{i}(\overline{\boldsymbol{\alpha}})\right|^{2} \\
{[\boldsymbol{F}(\overline{\boldsymbol{\alpha}})]_{k, l} } & =\frac{2}{\sigma^{2}} \sum_{i=1}^{m} \frac{\partial}{\partial \alpha_{k}} s_{i}(\overline{\boldsymbol{\alpha}}) \frac{\partial}{\partial \alpha_{l}} s_{i}(\overline{\boldsymbol{\alpha}})^{*}
\end{aligned}
$$

for $k, l \in\{1,2\}$.

Before we derive the FIM, we have to simplify the equation of $s_{i}(\overline{\boldsymbol{\alpha}})$ to be able to get a numerical solution for the derivatives. We assume that the phase shift is constant and is not involved in estimating the delay. This assumption causes some loss of information but is acceptable if the difference between the delays $\tau_{0}$ and $\tau_{1}$ is small. Phase difference information will still be included in the derivation by defining $A_{0}=a_{0} \mathrm{e}^{-\phi\left(\tau_{0}\right)}$ and $A_{1}=a_{1} \mathrm{e}^{-\phi\left(\tau_{1}\right)}$ as the complex amplitudes of the LOS and reflected signals. Therefore, the signal equation can be written as

$s_{i}(\overline{\boldsymbol{\alpha}})=A_{0} s[i] * \operatorname{sinc}\left(i-\tau_{0} / T_{s}\right)+A_{1} s[i] * \operatorname{sinc}\left(i-\tau_{1} / T_{s}\right)$.

The elements of the FIM can now be derived using (8) and (9):

$$
\begin{array}{r}
{[\boldsymbol{F}(\overline{\boldsymbol{\alpha}})]_{k, k}=} \\
{[\boldsymbol{F}(\overline{\boldsymbol{\alpha}})]_{k, l}=\frac{2}{\sigma^{2}} \sum_{i=1}^{m}\left|\frac{\partial}{\partial \tau_{k}} A_{k}^{m} s[i] * \operatorname{sinc}\left(i-\tau_{k} / T_{s}\right)\right|^{2}} \\
\frac{\partial}{\partial \tau_{k}} A_{k} s[i] * \operatorname{sinc}\left(i-\tau_{k} / T_{s}\right) \\
\cdot \frac{\partial}{\partial \tau_{l}} A_{l}^{*} s^{*}[i] * \operatorname{sinc}\left(i-\tau_{l} / T_{s}\right) .
\end{array}
$$

By defining

$r\left(\tau_{k}\right)=\frac{\partial}{\partial \tau_{k}} \operatorname{sinc}\left(i-\tau_{k} / T_{s}\right)=\frac{\sin \pi\left(i-\frac{\tau_{k}}{T_{s}}\right)}{\pi T_{s}\left(i-\frac{\tau_{k}}{T_{s}}\right)^{2}}-\frac{\cos \pi\left(i-\frac{\tau_{k}}{T_{s}}\right)}{T_{s}\left(i-\frac{\tau_{k}}{T_{s}}\right)}$,

we can easily obtain

$$
\begin{aligned}
& {[\boldsymbol{F}(\overline{\boldsymbol{\alpha}})]_{k, k}=\frac{2 a_{k}^{2}}{\sigma^{2}} \sum_{i=1}^{m}\left|s[i] * r\left(\tau_{k}\right)\right|^{2}} \\
& {[\boldsymbol{F}(\overline{\boldsymbol{\alpha}})]_{1,2}=\frac{2 a_{0} a_{1} \mathrm{e}^{j 2 \pi f_{c}(\Delta \tau)}}{\sigma^{2}} \sum_{i=1}^{m} s[i] * r\left(\tau_{0}\right) \cdot s^{*}[i] * r\left(\tau_{1}\right)} \\
& {[\boldsymbol{F}(\overline{\boldsymbol{\alpha}})]_{2,1}=\frac{2 a_{0} a_{1} \mathrm{e}^{-j 2 \pi f_{c}(\Delta \tau)}}{\sigma^{2}} \sum_{i=1}^{m} s[i] * r\left(\tau_{1}\right) \cdot s^{*}[i] * r\left(\tau_{0}\right),}
\end{aligned}
$$

where $\Delta \tau=\tau_{1}-\tau_{0}$.

In this paper, we are interested to study the estimation accuracy of $\tau_{0}$ only. Therefore, we want to calculate the value of the first diagonal element of the inverse of the FIM. The value is calculated and given in (13) in the bottom of this page.

$$
\left[\boldsymbol{F}^{-1}\right]_{1,1}=\frac{\sigma^{2} \sum_{i=1}^{m}\left|s[i] * r\left(\tau_{1}\right)\right|^{2}}{2 a_{0}^{2}\left(\sum_{i=1}^{m}\left|s[i] * r\left(\tau_{0}\right)\right|^{2} \sum_{i=1}^{m}\left|s[i] * r\left(\tau_{1}\right)\right|^{2}-\sum_{i=1}^{m} s[i] * r\left(\tau_{1}\right) \cdot s^{*}[i] * r\left(\tau_{0}\right) \sum_{i=1}^{m} s[i] * r\left(\tau_{0}\right) \cdot s^{*}[i] * r\left(\tau_{1}\right)\right)}
$$




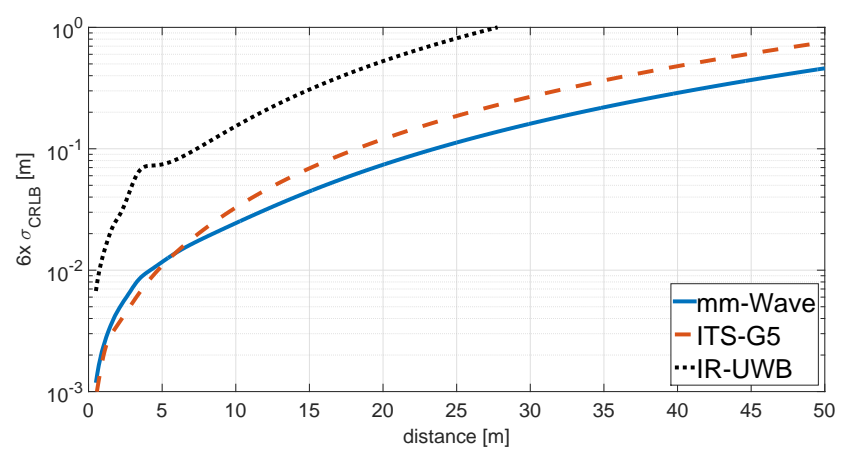

Fig. 3: Distance estimation lower bounds for mm-Wave, ITSG5, and IR-UWB signals.

As can be expected, if we consider each multipath component to be spaced long enough to avoid different pulses to overlap and if we assume the delays to be integers we will end up with the same lower bounds as derived in [4] and [5]. It should also be noted that if we only consider the LOS component and neglect the reflected signal, all the FIM components except $[\boldsymbol{F}(\overline{\boldsymbol{\alpha}})]_{1,1}$ will be equal to zero. The delay estimation accuracy in this case will be equal to $[\boldsymbol{F}(\overline{\boldsymbol{\alpha}})]_{1,1}^{-1}$.

\section{Simulation Results}

In this section, the CRLB of the ranging estimate between two nodes will be evaluated using the channel model suggested in Section II. The CRLB is calculated for a wide band $5 \mathrm{G}$ proposed signal operating in the $\mathrm{mm}$-Wave frequency band, an ITS-G5 OFDM signal, and an IR-UWB signal operating in the $6 \mathrm{GHz}$ frequency. The simulation parameters of each signal are summarized in Table 1. To provide a fair comparison, a realistic path loss model was implemented where oxygen absorption is accounted for mm-Wave propagation. Also, the amount of observed samples depends on the duration of observation $(0.1 \mathrm{~ms}$ in our case). Therefore, the amount of observed samples for mm-Wave and IR-UWB is fifty times higher compared to ITS-G5 due to the bandwidth difference.

As we are interested in analyzing the three chosen systems for ultra-reliable applications, it was decided to calculate the estimation accuracy lower bounds multiplied by a factor of six ( $6 \sigma$ figure-of-merit) which translates to reliability of $99.9997 \%$ and base our evaluation on the simulated results.

TABLE I: Simulation parameters

\begin{tabular}{|c||c|c|c|}
\hline & mm-Wave & ITS-G5 & IR-UWB \\
\hline Center Frequency & $60 \mathrm{GHz}$ & $5.9 \mathrm{GHz}$ & $6 \mathrm{GHz}$ \\
\hline EIRP & $31 \mathrm{dBm}$ & $31 \mathrm{dBm}$ & $-14.5 \mathrm{dBm}$ \\
\hline Pulse shape & Raised Cosine & OFDM & $\begin{array}{c}\text { Second-order } \\
\text { Gaussian monocycle }\end{array}$ \\
\hline BW & $500 \mathrm{MHz}$ & $10 \mathrm{MHz}$ & $500 \mathrm{MHz}$ \\
\hline Sampling period & $0.2 \mathrm{nsec}$ & $10 \mathrm{nsec}$ & $0.2 \mathrm{nsec}$ \\
\hline $\begin{array}{c}\text { Number of } \\
\text { observed samples }\end{array}$ & $500 \mathrm{k}$ & $10 \mathrm{k}$ & $500 \mathrm{k}$ \\
\hline
\end{tabular}

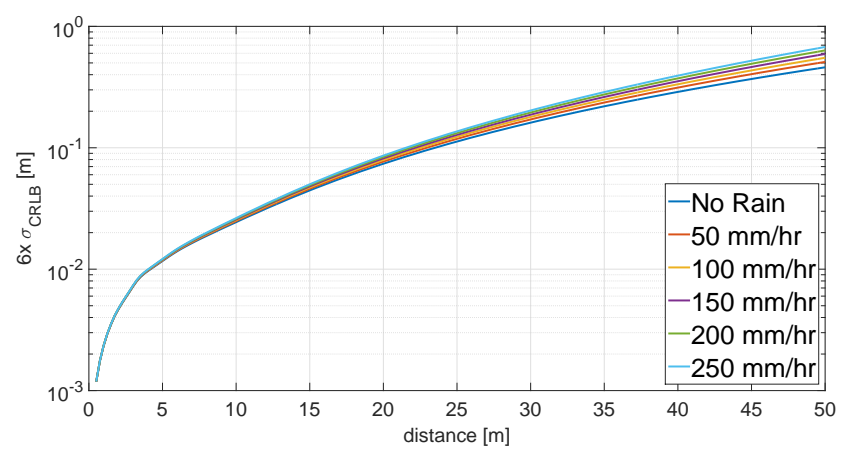

Fig. 4: Effect of rain attenuation on distance estimation lower bounds for mm-Wave signals.

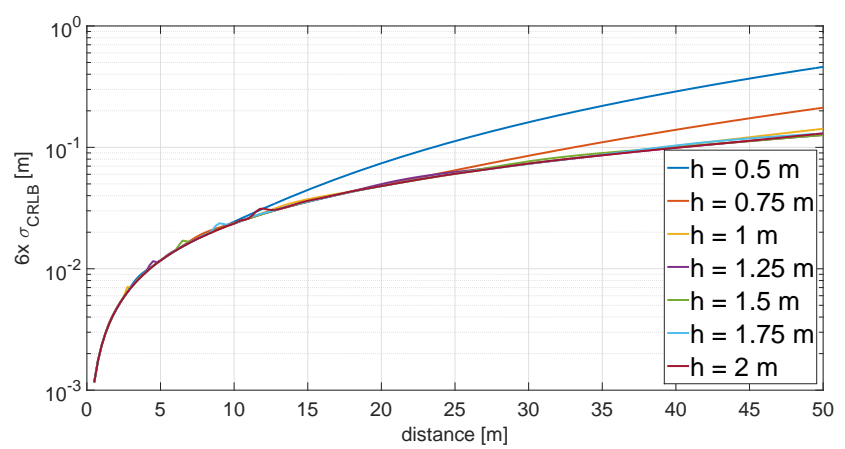

Fig. 5: Effect of changing antenna heights on distance estimation lower bounds for mm-Wave signals.

Figure 3 shows the distance estimation accuracy for transceivers having separation distances of up to 50 meters. The antenna heights to the ground surface $(h)$ is equal to 0.5 meter. We considered oversampling by a factor of ten. The geometry, delay, and path-loss are calculated for each separation distance, then the lower bounds are calculated using (16). The proposed mm-Wave signal outperforms both ITSG5 and IR-UWB due to the wider bandwidth compared to ITS-G5 and the higher transmit power compared to IR-UWB. It is also shown that the distance accuracy lower bounds are in the decimeter to meter range for distances around $50 \mathrm{~m}$ and it reaches millimeter to centimeter accuracy for distances of a few meters. These are acceptable accuracy figures for ultra-reliable applications provided by both $\mathrm{mm}$ Wave and ITS-G5 signals. But due to the wide bandwidth of the mm-Wave signal, it is easier to reach a close accuracy to the lower bounds in practical systems using the mm-Wave signal compared to ITS-G5 which will require more accurate interpolation and sophisticated receivers to reach the desired accuracy. The irregularities visible from both mm-Wave and IR-UWB curves in the separation distances between 3 and 4 meter occur because the LOS and reflected pulses start to overlap at these distances. For ITS-G5, the LOS and reflected pulses are always overlapping because the pulse width is fifty times longer.

Figure 4 shows the effect of rain on distance estimation 
accuracy using the proposed system operating in the mm-Wave frequency band. A model proposed in [13] was used. The rate of rain simulated varies from 0 (no rain) to the extreme 250 $\mathrm{mm} / \mathrm{hr}$. Loss due to rain with rate of $250 \mathrm{~mm} / \mathrm{hr}$ causes an extra loss of nearly $70 \mathrm{~dB} / \mathrm{km}$. This can cause severe performance degradation in large distances. But as noted from the results, rain has minimal effect in short distances.

Further, in Figure 5, the effect of antenna heights on distance estimation accuracy is simulated. It is shown that the performance is worse at small heights. When heights increase to 1 meter and higher the performance is enhanced. The reason is that the effect of the reflected multipath signal on the LOS signal is decreased. At antenna heights 1 meter and more, the performance is almost the same as the signals from the LOS and reflected paths are almost not overlapping anymore and could be treated separately.

\section{CONCLUSION}

In this paper, we proposed a LOS geometry-based channel model for the scenario of automatic coupling in the railway domain. We derived a suitable discrete equivalent system representation that takes into account non-integer delay and used the representation to derive CRLB for delay estimation accuracy. The lower bounds were used to compare between system candidates for ultra-reliable communications and ranging in the railway domain. The three systems are ITS-G5, IR-UWB, and a proposed wide band $5 \mathrm{G} \mathrm{mm-Wave} \mathrm{system.}$ Results show that the proposed mm-Wave system outperforms the other systems in ranging accuracy at short distances up to 50 meter. The results also show that mm-Wave provides good ranging accuracy at severe rainy weather. Antenna heights of 1 meter and higher to the ground surface provide the best ranging accuracy for the proposed $5 \mathrm{G} \mathrm{mm}$-Wave system.

\section{ACKNOWLEDGMENT}

Part of this work has been performed in the framework of the BMBF project SBDist [14]. The authors would like to acknowledge the contributions of their colleagues, although the views expressed are those of the authors and do not necessarily represent the project.

\section{REFERENCES}

[1] P. Popovski, "Ultra-reliable communication in 5G wireless systems," 1st International Conference on 5G for Ubiquitous Connectivity, Akaslompolo, 2014, pp. 146-151.

[2] P. Kumari, N. Gonzalez-Prelcic and R. W. Heath, "Investigating the IEEE 802.11ad Standard for Millimeter Wave Automotive Radar," 2015 IEEE 82nd Vehicular Technology Conference (VTC2015-Fall), Boston, MA, 2015, pp. 1-5.

[3] Van Trees, Harry L. Detection, estimation, and modulation theory. John Wiley \& Sons, 2004.

[4] Lei Huang and Chi Chung Ko, "Performance of maximum-likelihood channel estimator for UWB communications," in IEEE Communications Letters, vol. 8, no. 6, pp. 356-358, June 2004.

[5] S. Khalesehosseini and J. Nielsen, "Cramer-Rao lower bound for dataaided and non-data-aided synchronisation of ultra-wideband signals with clock offset," in IET Communications, vol. 3, no. 1, pp. 135-142, January 2009.
[6] R. Cardinali, L. De Nardis, P. Lombardo and M. G. Di Benedetto, "Lower bounds for ranging accuracy with multi band OFDM and direct sequence UWB signals," 2005 IEEE International Conference on UltraWideband, 2005, pp. 302-307.

[7] L. Huang and C. C. Ko, "Cramer-Rao Lower Bounds for Semi-Blind ML Channel Estimation in UWB Systems," in IEEE Transactions on Wireless Communications, vol. 5, no. 12, pp. 3388-3393, December 2006.

[8] Y. Shoji, H. Sawada, C. S. Choi and H. Ogawa, "A Modified SV-Model Suitable for Line-of-Sight Desktop Usage of Millimeter-Wave WPAN Systems," in IEEE Transactions on Antennas and Propagation, vol. 57, no. 10 , pp. 2940-2948, Oct. 2009.

[9] IEEE P802.15.3c/D08. Part 15.3: Wireless Medium Access Control (MAC) and Physical Layer (PHY) Specifications for High Rate Wireless Personal Area Networks (WPANs): Amendment 2: Millimeter-wave based Alternative Physical Layer Extension, March 2009.

[10] IEEE 802.11ad Part 11: Wireless LAN Medium Access Control (MAC) and Physical Layer (PHY) Specifications: Amendment 3: Enhancements for Very High Throughput in the $60 \mathrm{GHz}$ Band, 2012.

[11] L. Cui, H. Zhang, X. Liu and T. A. Gulliver, "A ranging method for $60 \mathrm{GHz}$ OFDM system based on improved preamble sequence," 2015 IEEE Pacific Rim Conference on Communications, Computers and Signal Processing (PACRIM), Victoria, BC, 2015, pp. 246-251.

[12] Alan, V. Oppenheim, W. Schafer Ronald, and R. B. John. 'Discrete-time signal processing." New Jersey, Printice Hall Inc (1989).

[13] Recommendation ITU-R P.838-2, "Specification attenuation model for rain for use in prediction methods", 2003.

[14] "SBDist," $2015 . \quad$ [Online]. Available: http://www.industrialradio.de/Projects/Home/SBDist 